\title{
Propiedades antimicrobianas y citotóxicas de un adhesivo de uso ortodóncico adicionado con nanopartículas de plata'
}

\section{Antimicrobial and cytotoxic properties of an orthodontic adhesive added with silver nanoparticles applications}

\author{
Liliana Argueta-Figueroa, ${ }^{*}$ Ma. Concepción Arenas-Arrocena, ${ }^{*}$ \\ Ana Paulina Díaz-Herrera, ${ }^{* *}$ Susana Vanessa García-Benítez, ${ }^{* *}$ \\ René García-Contreras*
}

\begin{abstract}
The objective of this study was to evaluate the antibacterial effect and cytotoxicity of an orthodontic adhesive with silver nanoparticles (Ag NP's). The antimicrobial activity was determined by a disc diffusion test for Staphylococcus aureus, Escherichia coli, and Enterococcus faecalis at different concentrations of silver nanoparticles in an orthodontic adhesive. As well as cytotoxicity of said adhesive to be incorporated Ag NP's was evaluated, through the MTT method. The results showed that the bacteria under study, S. aureus, E. coli and E. faecalis were susceptible both to the Ag NP's and to the orthodontic MIP adhesive added with Ag NP's at 10, 5 and 3 v/v\%. The antibacterial effect of silver nanoparticles is dose dependent. The cytotoxicity of the orthodontic MIP adhesive itself exhibits a mild to moderate cytotoxicity as well as incorporating the Ag NP's at the concentrations above mentioned. The nanoparticles incorporated in the adhesive for orthodontic use show antibacterial effect as well as maintain a slight cytotoxicity.
\end{abstract}

KEYWORDS: biocompatibility, cell viability, nanotoxicology, antibacterial effect, metal nanoparticles, orthodontic adhesive.

RESUMEN: El objetivo de este estudio fue evaluar el efecto antibacteriano y la citotoxicidad de un adhesivo de uso ortodóncico con nanopartículas de plata (Ag NP's). Se determinó la actividad antimicrobiana mediante una prueba de difusión en disco para Staphylococcus aureus, Escherichia coli, y Enterococcus faecalis a diferentes concentraciones de nanopartículas de plata en un adhesivo ortodóncico. También se evaluó la citotoxicidad de dicho adhesivo al ser incorporadas

Recibido: 16 de junio de 2017.

Aceptado: 10 de abril de 2018.

- Agradecimientos: Liliana Argueta-Figueroa agradece a la DGAPA-UNAM por la beca posdoctoral brindada. Gracias por el financiamiento otorgado a través de PAPIIT IN225516 y IA204516. PAPIME: PE210616, PE201617 y PE208518; Sener-Conacyt proyecto no. 207450 (cEMIE-Sol/27), Conacyt-México (cB176450) y Sener-Conacyt (245811), y Red Farmoquímicos de Conacyt.

* Universidad Nacional Autónoma de México. Laboratorio de Investigación Interdisciplinaria, Área de Nanoestructuras y Biomateriales, Escuela Nacional de Estudios Superiores Unidad León; León, Guanajuato, México

** Universidad Nacional Autónoma de México. Escuela Nacional de Estudios Superiores Unidad León; León, Guanajuato, México

Autor de correspondencia: (dentist.garcia@gmail.com). 
Ag NP's, mediante el método de MTT. Los resultados mostraron que las bacterias en estudio, S. aureus, E. coli y E. faecalis fueron susceptibles tanto a las Ag NP's como al adhesivo MIP ortodóncico adicionado con Ag NP's a concentraciones de 10, 5 y 3 v/v\%.

El efecto antibacteriano de las nanopartículas de plata es dosis dependiente. La citotoxicidad del adhesivo MIP ortodóncico en sí mismo exhibe una citotoxidad ligera a moderada, así como al incorporarle las Ag NP's a las concentraciones antes mencionadas. Las nanopartículas incorporadas en el adhesivo de uso ortodóncico muestran efecto antibacterial así como mantienen una citotoxicidad ligera.

PALABRAS CLAVE: biocompatibilidad, viabilidad celular, nanotoxicología, efecto antimicrobiano, nanopartículas metálicas, adhesivo ortodóncico.

\section{Introducción}

Existen en el mercado diferentes sistemas de adhesión para la fijación de brackets en ortodoncia, entre los cuales se encuentran los sistemas con primer-adhesivo hidrofílicos (MIP) y sistemas de resina hidrófila que se han introducido recientemente en la práctica odontológica, los cuales pretenden proporcionar una mejor adhesión al esmalte (Anand et al., 2014). El MIP más comúnmente utilizado es Transbond MIP (3M Unitek, Monorovia, California, EUA) Transbond MIP permite la adhesión en un entorno húmedo sin comprometer su fuerza de adhesión. Se han publicado numerosos estudios in vitro sobre la efectividad de este adhesivo (Littlewood et al., 2000; Webster et al., 2001; Anand et al., 2014; Deng et al., 2016; Grandhi y Combe, 2001).

A pesar de los grandes avances científicos en materiales adhesivos utilizados en ortodoncia, se necesitan mejoras adicionales (Argueta-Figueroa et al., 2015) para evitar la formación indeseable de la lesiones de mancha blanca en el esmalte (Sudjalim y Woods, 2006). La desmineralización del esmalte es común durante el tratamiento de ortodoncia fija y se asocia con la acumulación de placa dental retenida alrededor de la aparatología y los agentes de unión (adhesivo y resina) (Heymann y Grauer, 2013). Estudios previos han demostrado que hay un aumento significativo de bacterias causantes de caries cuando se colocan aparatos ortodóncicos fijos. Generalmente, la producción de ácido por bacterias causa la desmineralización de la superficie del esmalte, lo que puede conducir a caries dentales (Sukontapatipark et al., 2001). Una propiedad deseable de la ortodoncia adhesiva contemporánea es que pueda poseer un efecto antibacteriano que limite la proliferación bacteriana.

La aplicación de la nanotecnología en ciencia de materiales es un gran paso hacia la producción de materiales con mejores propiedades químicas, mecánicas, ópticas y eléctricas. Actualmente, una potencial aplicación de la nanotecnología es el efecto antibacteriano que pueden exhibir algunas nanopartículas (Hajipour et al., 2012), entre estas, destacan las nanopartículas de plata (Ag NP's) por su excelente efecto bactericida (García-Contreras et al,. 2011). Es un hecho bien conocido que los iones de plata y los compuestos a base de plata son altamente tóxicos para los microrganismos. 
Este aspecto de la plata lo convierte en una excelente opción para múltiples funciones en el área biomédica. La plata se usa generalmente en forma de nitrato para inducir el efecto antimicrobiano, pero cuando la plata alcanza la escala nanométrica, su efecto antimicrobiano aumenta. Se ha afirmado que la concentración de Ag NP's que impide el crecimiento bacteriano es dosis-dependiente y es diferente para cada tipo de bacteria (Souza et al., 2016; Prabhu y Poulose, 2012; Van Dong y Ha, 2012). También se ha investigado la actividad antimicrobiana de las Ag NP's y se ha concluido que la concentración mínima inhibitoria y mínima bactericida es diferente en principio por el tipo de membrana de las bacterias Gram positivas o Gram negativas (Franci et al., 2015; Hajipour et al,. 2012).

Es importante señalar que la acción antibacteriana de las Ag NP's es altamente dependiente del tamaño de la partícula, morfología y dosis (GarcíaContreras et al., 2011). Las NP's deben de ser lo suficientemente pequeñas para penetrar la membrana celular y con ello afectar la homeostasis intracelular. La interacción de las partículas con la bacteria depende del área superficial disponible (partículas pequeñas con una mayor área superficial disponible para la interacción tienen más efecto bactericida que las partículas grandes). Es posible que las Ag NP's no solo interactúen con la superficie de la membrana, sino que también penetren al interior de la bacteria (Xiu et al. 2012). De tal manera, el efecto bactericida de las Ag NP's puede ser un sinergismo de los siguientes mecanismos: las Ag NP's penetran a la bacteria con facilidad debido a su tamaño diminuto, las Ag NP's liberan iones de plata y se enlazan con grupos sulfhidrilos de biomoléculas y con compuestos fosforosulfurados como los presentes en el ADN, lo que inactiva a las bacterias y la producción de especies reactivas de oxígeno que causan estrés oxidativo que conlleva a la muerte bacteriana (Prabhu y Poulose, 2012).

Por lo tanto, con la finalidad de disminuir las complicaciones a consecuencia del tratamiento ortodóncico sin causar citotoxicidad, el propósito de este estudio fue la incorporación de nanopartículas de plata (Ag NP's) en un adhesivo MIP para determinar el efecto antibacteriano, así como evaluar la citotoxicidad del mismo. La hipótesis de trabajo es que el adhesivo adicionado con Ag NP's presentará efecto antibacteriano y citotoxicidad ligera.

\section{Material y método}

\section{Sintesis de Ag NP's}

La síntesis de las Ag NP's, utilizada en este estudio, se basó en el método desarrollado por Solomon et al. (2007), con algunas modificaciones. En una reacción típica, en $30 \mathrm{ml}$ de una solución recién preparada $0.002 \mathrm{M}$ de borohidruro de sodio $\left(\mathrm{NaBH}_{4}\right)$, se le agregaron $2 \mathrm{ml}$ de una solución $0.001 \mathrm{M}$ de nitrato de plata $\left(\mathrm{AgNO}_{3}\right)$ con una velocidad de adición de $0.2 \mathrm{ml} / \mathrm{seg}$. La disolución se mantuvo en agitación magnética hasta que se añadió completamen- 
te el $\mathrm{AgNO}_{3}$. La formación de las Ag NP’s puede confirmase al observar que la disolución presenta una coloración amarillo claro (Solomon et al. 2007).

\section{Preparación de las muestras de adhesivo adicionado con Ag NP's}

Para este estudio, se seleccionó un adhesivo ortodóncico fotopolimerizable convencional (Transbond MIP, 3M Unitek, CA, EUA) debido a sus propiedades hidrofílicas. Las Ag NP's, suspendidas en agua deionizada en una concentración de $3.3 \mathrm{nM}$, se añadieron al adhesivo con una micropipeta en diferentes concentraciones y se mezclaron en el adhesivo a través de agitación en vórtex por $1 \mathrm{~min}$. Los grupos de tratamiento que se emplearon en este estudio pueden observarse en la tabla 1.

\section{Prueba de difusión en disco}

La actividad antibacteriana del adhesivo se determinó por la técnica de difusión en disco de acuerdo con las recomendaciones del National Committee for Clinical Laboratory Standards. Se prepararon cultivos frescos con S. aureus, E. coli y $S$. faecalis. Los cultivos fueron ajustados con solución salina estéril para obtener una turbidez equivalente al 0.5 del estándar de McFarland. Se prepararon placas de agar Mueller-Hinton (MHA) y se inocularon con $200 \mu \mathrm{l}$ de cada cepa bacteriana del estándar de McFarland en dilución 1:20. Posteriormente se colocaron discos de papel filtro estériles sobre la superficie de las placas de agar y se agregaron con micropipeta $20 \mu \mathrm{l}$ del agente a probar en cada disco según correspondiera, se utilizó como control positivo (+) un disco con clorhexidina y como control negativo (-) se empleó un disco con agua destilada estéril; y se incubaron a $37^{\circ} \mathrm{C}$ durante 24 horas en condiciones aeróbicas. El ensayo se realizó por triplicado en dos ensayos independientes. Finalmente, se tomaron fotografías de las cajas y se midieron en milímetros los halos de inhibición bacteriana producidos en las placas de agar, con el programa Image J (U. S. National Institutes of Health, Bethesda, Maryland). El programa fue calibrado usando una distancia conocida y cada halo fue medido en tres direcciones, obteniéndose el promedio del diámetro de cada uno de los halos.

TABLA 1. Relación de adhesivo adicionado con Ag NP's en los grupos de tratamiento.

\begin{tabular}{l|c|c}
\multirow{2}{*}{ Grupos } & \multicolumn{2}{|c}{ Relación en porcentaje de volumen/volumen (v/v\%) } \\
\cline { 2 - 3 } Pg NP's & Porcentaje de adhesivo & Porcentaje de Ag NP's \\
\hline Adh/Ag NP's 10 & 0 & 100 \\
\hline Adh /Ag NP's 5 & 90 & 10 \\
Adh /Ag NP's 3 & 95 & 5 \\
\hline Adh & 97 & 3 \\
\hline
\end{tabular}

Fuente: Elaboración de los autores. 


\section{Ensayo de citotoxicidad}

Cultivo celular. Fibroblastos gingivales humanos (FGH) fueron obtenidos a partir de tejido gingival recolectado durante la extracción de la cirugía del tercer molar de un paciente de 25 años con consentimiento informado escrito previo firmado y aprobado por el Comité de Bioética de la Escuela Nacional de Educación Superior (ENES), Campus León, Universidad Nacional Autónoma de México (UNAM). Todos los tejidos se cortaron en pequeños explantes utilizando una hoja de bisturí estéril y los fragmentos de tejido se sembraron en una placa de cultivo de $10 \mathrm{~cm}$ y se cultivaron en Alpha Modification of Eagle's Medium ( $\alpha$-MEM, Life Technologies, Gibco, Carlsbad, CA, EUA) Complementado con un $20 \%$ de suero fetal bovino inactivado por calor (FBS, Life Technologies, Gibco), $100 \mathrm{UI} / \mathrm{ml}$ de penicilina, $100 \mathrm{mg} / \mathrm{ml}$ de estreptomicina (Life Technologies, Gibco). Los cultivos primarios se incubaron a $37{ }^{\circ} \mathrm{C}$ en una atmósfera humidificada de $\mathrm{CO}_{2}$ al $5 \%$ hasta que la población celular cubrió el $80 \%$ de la placa. Después, las células se recuperaron mediante tratamiento con $0.25 \%$ de tripsina-EDTA-2Na al $0.025 \%$ en PBS (-) y se subcultivaron o se usaron para experimentos. Las células se subcultivaron en medio de Eagle modificado de Dulbecco (DMEM, Life Technologies, Gibco) suplementado con $10 \%$ de SFB, $1 \%$ de Glutamax (Life Technologies, Gibco) y $2 \%$ de penicilina y estreptomicina (Life Technologies, Gibco).

Actividad citotóxica (dosis-respuesta). De un cultivo de fibroblastos gingivales humanos (FGH) en su división celular (PDL, del inglés population doubling level) 12 , se obtuvo un cultivo secundario en su división número 13. Se inocularon dichas células $\left(2 \times 10^{5}\right.$ células $\left./ \mathrm{ml}\right)$ en cada pocillo de una microplaca de 96 pocillos y se incubaron durante $24 \mathrm{~h}$ para lograr la completa confluencia y adherencia celular. Posteriormente, se inocularon las muestras de adhesivos con $\mathrm{Ag}$ NP's a diferentes concentraciones $(10,5,3$ y $0 \mathrm{v} / \mathrm{v} \%)$ previamente diluidas cada una de ellas en medio de cultivo en una relación 1:1 para permitir el crecimiento celular; más adelante se realizaron diluciones seriadas de cada una de las muestras. Después, se incubaron durante 24 horas a $37{ }^{\circ} \mathrm{C}$, con una humedad relativa del $95 \%$ y $\mathrm{CO}_{2}$ al $5 \%$. El número relativo de células viables se determinó mediante el método MTT (Sigma-Aldrich, St Luis, MO, EUA). El medio de cultivo de cada pocillo se reemplazó con MTT $(0.2 \mathrm{mg} / \mathrm{ml})$ disuelto en DMEM, y las células se incubaron durante $4 \mathrm{~h}$ a $37^{\circ} \mathrm{C}$. Después de reemplazar el medio, el formazano se disolvió completamente en dimetilsulfóxido (DMSO, SigmaAldrich) y se determinó la absorbancia a $570 \mathrm{~nm}$ utilizando un lector de microplacas (Thermo Scientific, St. Luis, MO, EUA). La citotoxicidad se calificó de acuerdo con la norma ISO 10993-5 como no citotóxica (viabilidad celular superior al $75 \%$ ), ligeramente citotóxica (viabilidad celular entre $50 \%$ a $75 \%$ ), moderadamente citotóxica (la viabilidad celular entre $25 \%$ a $50 \%$ ), y severamente citotóxica (viabilidad celular inferior al 25\%). La concentración citotóxica media $\left(C_{50}\right)$ se determinó a partir de la curva dosis-respuesta. Se obtuvieron datos reproducibles por triplicado y en tres experimentos independientes. 


\section{Análisis estadístico}

Los datos descriptivos se expresan como la media \pm desviación estándar (SD). La prueba de normalidad de Shapiro-Wilk se realizó para todos los datos. El análisis estadístico se realizó mediante la prueba de ANOVA de una vía, t de Student, Kruskal-Wallis y la prueba U de Mann-Whitney. Se utilizó SPSS (Paquete estadístico para las ciencias sociales, Chicago, IL, EUA) En todos los análisis. Las diferencias se consideraron significativas si $p \leq 0.05$.

\section{Resultados y discusión}

\section{Sintesis de Ag NP's}

En la figura 1, se observa el pico de la resonancia de plasmón superficial en $399 \mathrm{~nm}$, el cual se encuentra dentro del rango característico de la banda de absorción centrada en 395 a $438 \mathrm{~nm}$ asignada a la resonancia del plasmón superficial de nanopartículas de plata de forma esférica, el tamaño de estas es mayor conforme más se encuentra desplazado a la derecha de $400 \mathrm{~nm}$ del espectro. La anchura a media altura (Full Width at Half Maximum, FWHM) del plasmón fue de $68 \mathrm{~nm}$. De acuerdo con un reporte de Solomon, et al., 2007, con estos datos puede predecirse el tamaño de partícula de las Ag NP's (a través de este método de síntesis a través de reducción química) el cual se encuentra en el rango de 10-14 nm de diámetro.

En este mismo estudio se postula que la adsorción de borohidruro juega un papel clave en la estabilización de las nanopartículas de plata durante su formación al proporcionar una carga negativa superficial de partículas. Los átomos de plata que conforman cada nanopartícula son pasivados o estabilizados por los aniones del [BH-] provenientes del agente reductor, un diagrama ilustrativo de lo anterior puede observarse en la figura 2. Por lo tanto,

FIGURA 1. Espectro de Uv-vis de las nanopartículas de plata.

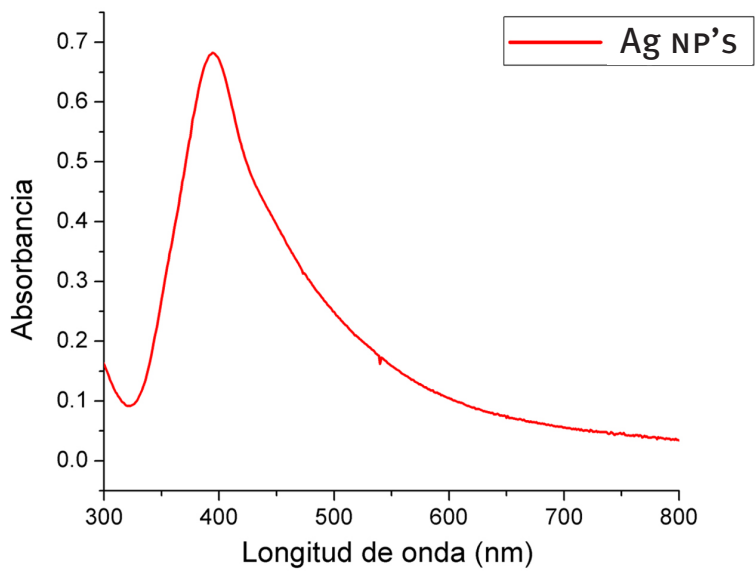

Fuente: Elaboración de los autores. 
FIGURA 2. Diagrama ilustrativo de la pasivación de una nanopartícula de plata con los aniones [BH-] provenientes del borohidruro de sodio.

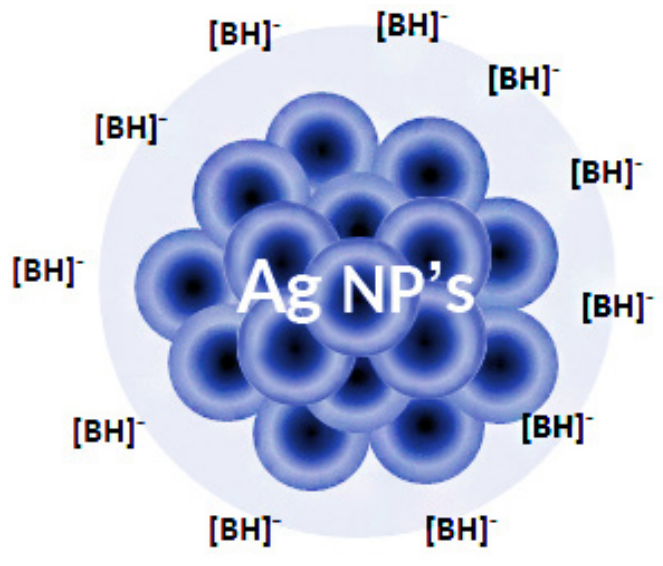

Fuente: Elaboración de los autores.

mientras la proporción de borohidruro de sodio: nitrato de plata se encuentre en una relación estequiométrica de 2:1, las partículas obtenidas serán estables a través del tiempo (Solomon et al., 2007) y esto se confirma a través del espectro de Uv-vis. En el método de síntesis empleado por Solomon se propone que con el fin de evitar la precipitación o agregación de las partículas se adicione un surfactante, polivinilpirrolidona (PVP), sin embargo, en este estudio no se utilizó dicho surfactante pues se colocaron en el adhesivo y este cumplió con la función de estabilizar a las NP's.

\section{Prueba de susceptibilidad antimicrobiana por difusión en agar}

Entre las complicaciones más importantes del tratamiento de ortodoncia fija se encuentra la desmineralización del esmalte, ya que la aparatología fija ortodóncica facilita la acumulación de placa, lo que conlleva a un aumento en el recuento bacteriano (Agarwal et al,. 2015), debido a esto, es importante desarrollar un adhesivo que presente actividad antimicrobiana para reducir la formación de biofilm en la interfase diente-bracket.

La prueba de difusión en agar determina la capacidad de los agentes antibacterianos para difundirse con el agar y producir una zona de inhibición bacteriana (Rose y Miller, 1939), sin embargo, es una prueba cualitativa que solo puede mostrar si existe o no un efecto antimicrobiano (Jorgensen y Turnidge, 2015), cuando no ha sido estandarizado a través de regresión lineal la asociación entre agentes antimicrobianos nuevos y la susceptibilidad a cada cepa bacteriana (Matuschek, Brown y Kahlmeter, 2014). Por otro lado, esta prueba es útil para detectar resistencias bacterianas (Balouiri y Sadiki, 2016), esto es evidente cuando se observan colonias dentro de los límites de la zona de inhibición. En la presente prueba de difusión en agar de este estudio, no se observaron colonias que indicaran resistencia bacteriana en ninguna de las cepas en 
estudio. Por otra parte, los promedios de los halos de inhibición se compararon por medio de la prueba estadística de Mann-Whitney, entre las diferentes concentraciones de las nanopartículas de plata incorporadas en el adhesivo ortodóncico. En la tabla 2 se muestran los resultados de dicha prueba y las comparaciones múltiples. Se observa que las Ag NP's (sin adhesivo) son las que exhiben para todas las cepas bacterianas estudiadas el mejor efecto antibacterial, y que dicho efecto se ve ligeramente disminuido al incorporarse en el adhesivo. También los resultados de esta prueba muestran que el efecto es dosis-dependiente, es decir, que mientras mayor es la dosis de las Ag NP's incorporadas, mejor es el efecto antibacteriano que exhibe el adhesivo.

\section{Ensayo de citotoxicidad}

En la tabla 3 y figura 3 se muestran los resultados obtenidos de la viabilidad celular relativa en FGH expuestos a diferentes concentraciones de adhesivo ortodóncico adicionado con nanopartículas de plata (v/v\%). Las nanopartículas por sí solas son consideradas no citotóxicas (viabilidad celular $\geq 75 \%$ ) excepto en las tres dosis más altas en donde resultaron tener una citotoxicidad ligera (viabilidad celular $<75 \%$ ), el adhesivo sin nanopartículas muestra una citotoxidad severa (viabilidad celular $<25 \%$ ) en la dosis más alta. La citotoxicidad de los compuestos correspondió de la siguiente manera con base en la concentración citotóxica media $\left(\mathrm{CC}_{50}\right)$ : Adh/Ag NP's $5(0.95 \%)>\mathrm{Adh}$ (1.37\%)>Adh/Ag NP's 3 (1.45\%)>Adh/Ag NP's 10 (1.48\%). El análisis estadístico se llevó a cabo a través de comparaciones múltiples con t de Student y Post ANOVA de Tukey.

A pesar de que las nanopartículas de plata han sido muy estudiadas siguen siendo una de las áreas de investigación más controvertidas con respecto a su toxicidad para los sistemas biológicos. Se ha encontrado que in-

TABLA 2. Halos de inhibición producidos en la prueba de susceptibilidad antimicrobiana por difusión en agar.

\begin{tabular}{l|c|c|c|}
\multirow{2}{*}{} & \multicolumn{3}{|c|}{ Halo de inhibición (mm) } \\
\cline { 2 - 4 } & S. aureus & E. coli & E. faecalis \\
\hline Ag NP's & $16^{\mathrm{a}}$ & $15^{\mathrm{a}}$ & $15^{\mathrm{a}}$ \\
\hline Adh/Ag NP's 10 & $14^{\mathrm{a}}$ & $12^{\mathrm{b}}$ & $12^{\mathrm{b}}$ \\
\hline Adh/Ag NP's 5 & $15^{\mathrm{a}}$ & $8^{\mathrm{c}}$ & $12^{\mathrm{b}}$ \\
\hline Adh/Ag NP's 3 & $10^{\mathrm{b}}$ & $14^{\mathrm{a}}$ & $10^{\mathrm{b}}$ \\
\hline Adh & $0^{\mathrm{c}}$ & $0^{c}$ & $0^{c}$ \\
\hline Control (+) & $24^{\mathrm{d}}$ & $22^{\mathrm{d}}$ & $19^{\mathrm{d}}$ \\
\hline Control (-) & $0^{\mathrm{c}}$ & $0^{c}$ & $0^{c}$ \\
\hline
\end{tabular}

Letras minúsculas diferentes son estadísticamente significativas a la comparación intra grupos (U de Mann-Whitney, $p<0.05)$. Letras iguales no muestra diferencias entre los grupos $(p>0.05)$.

Fuente: Elaboración de los autores. 
TABLA 3. Halos de inhibición producidos en la prueba de susceptibilidad antimicrobiana por difusión en agar.

\begin{tabular}{|c|c|c|c|c|c|}
\hline $\begin{array}{c}\text { Concentración } \\
\qquad(\%)\end{array}$ & Ag NP's & $\begin{array}{l}\mathrm{Adh} / \mathrm{Ag} \\
\text { NP's } 10\end{array}$ & $\begin{array}{l}\text { Adh/Ag } \\
\text { NP's } 5\end{array}$ & $\begin{array}{l}\mathrm{Adh} / \mathrm{Ag} \\
\text { NP's } 3\end{array}$ & Adh \\
\hline 0 & $100 \pm 9.2^{\mathrm{aA}}$ & $100 \pm 12.7^{\mathrm{aA}}$ & $100 \pm 11.5^{\mathrm{aA}}$ & $100 \pm 8.4^{\mathrm{aA}}$ & $100 \pm 7.9^{\mathrm{aA}}$ \\
\hline 0.78125 & $78.93 \pm 3.9^{\mathrm{bA}}$ & $50.15 \pm 5.7^{\mathrm{bB}}$ & $45.41 \pm 5.3^{\mathrm{bB}}$ & $49.98 \pm 3.4^{\mathrm{bB}}$ & $49.32 \pm 5.6^{\mathrm{bB}}$ \\
\hline 1.5625 & $84.02 \pm 3.5^{\mathrm{bA}}$ & $41.06 \pm 4.5^{\mathrm{cB}}$ & $43.72 \pm 4.7^{\mathrm{bB}}$ & $51.4 \pm 5.8^{\mathrm{bc}}$ & $51.4 \pm 5.8^{\mathrm{bc}}$ \\
\hline 3.125 & $82.13 \pm 2.9^{\mathrm{bA}}$ & $48.31 \pm 4.7^{\mathrm{bB}}$ & $48.89 \pm 3.2^{\mathrm{bB}}$ & $44.68 \pm 3.3^{b c}$ & $49.22 \pm 4.3^{\mathrm{bB}}$ \\
\hline 6.25 & $80.87 \pm 4.4^{\mathrm{bA}}$ & $55.39 \pm 5.4^{\mathrm{bB}}$ & $57.58 \pm 4.7^{\mathrm{CB}}$ & $48.05 \pm 3.1^{b c}$ & $47.93 \pm 11.9^{b c}$ \\
\hline 12.5 & $74.91 \pm 5.3^{\mathrm{cA}}$ & $59.46 \pm 4.8^{\mathrm{bB}}$ & $58.17 \pm 5.3^{\text {св }}$ & $41.22 \pm 5.6^{c c}$ & $43.1 \pm 5.02^{\mathrm{bc}}$ \\
\hline 25.0 & $70.82 \pm 4.4^{\mathrm{cA}}$ & $45.32 \pm 5.9^{\mathrm{cB}}$ & $41.45 \pm 4.7^{\mathrm{bB}}$ & $33.45 \pm 3.6^{d c}$ & $27.98 \pm 11.9^{d C}$ \\
\hline 25.0 & $64.46 \pm 9.4 \mathrm{dA}$ & $29.47 \pm 5.4^{\mathrm{dB}}$ & $18.17 \pm 5.3^{\mathrm{dc}}$ & $11.99 \pm 3.5^{\mathrm{eD}}$ & $5.79 \pm 6.7^{\mathrm{eE}}$ \\
\hline
\end{tabular}

Letras minúsculas diferentes son estadísticamente significativas a la comparación intra grupos $(t-s t u d e n t$ pareada, $p \leq 0.05)$. Letras mayúsculas diferentes son estadísticamente significativas diferentes a la comparación entre grupos a la misma dosis (Post ANOVA, $p \leq 0.05$ ). Letras iguales no muestran diferencias estadísticamente significativas entre los grupos ( $p>0.05)$.

Fuente: Elaboración de los autores.

ducen citotoxicidad moderada in vitro en ensayos de micronúcleos en células TK6 (Li et al., 2012) y células de hígado de rata BRL 3a (Hussain et al., 2005). En otro estudio se analizó la distribución de las Ag NP's al estar en contacto con células y estas se encontraron dentro de las mitocondrias y el núcleo empleando microscopía electrónica de transmisión (TEM), lo cual implica su

FIGURA 3. Gráfico de viabilidad celular de FGH en contacto con el adhesivo de uso ortodóncico adicionado con nanopartículas de plata a diferentes concentraciones.

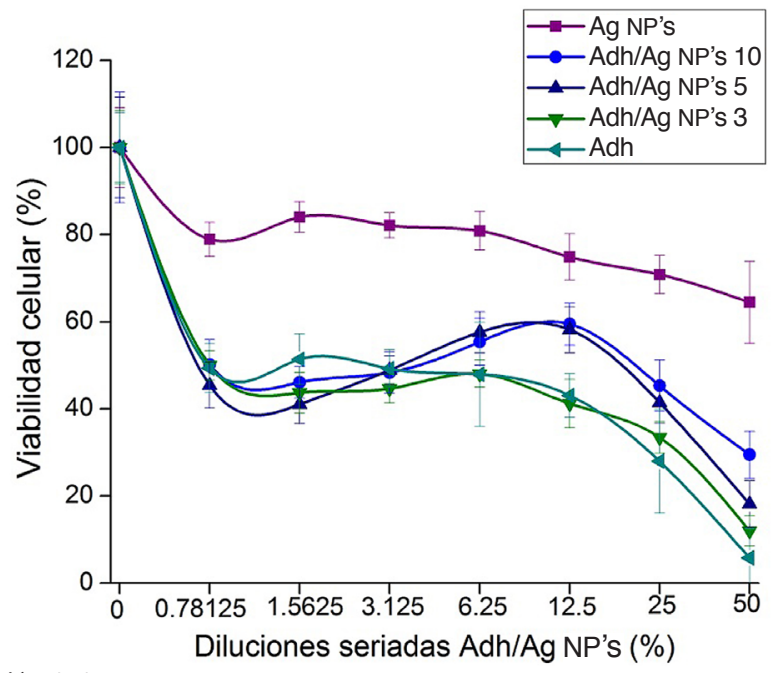

Fuente: Elaboración de los autores. 
participación directa en la toxicidad mitocondrial y el daño del ADN. En dicho estudio se propuso un posible mecanismo de toxicidad que implica la interrupción de la cadena respiratoria mitocondrial por las Ag NP's, lo que conduce a la producción de especies reactivas al oxígeno (ROS, por sus siglas en inglés) e interrupción de la síntesis de ATP, que a su vez causa daño en el ADN. Se anticipa que el daño en el ADN se ve aumentado por la deposición de las NP's, seguido de las interacciones de Ag NP's con el ADN, lo que conduce al detenimiento del ciclo celular en la fase G2/M (Choi y Hu, 2008). Asimismo, se ha hipotetizado que la liberación de iones extracelular e intracelularmente es un mecanismo que actúa de forma simultánea a la deposición de dichas nanopartículas (Choi et al. 2008). También se ha postulado que otro posible mecanismo de muerte celular es a través de apoptosis mediada por vía receptor 2 tipo-Toll (Kim et al., 2012), un diagrama de estos mecanismos puede observarse en la figura 4. Por otro lado, los resultados de un estudio in vivo realizado por Kim et al. (2008) mostraron que las nanopartículas de plata no afectaron ni el conteo de eritrocitos policromáticos micronucleados, tomados como un indicador de daño en el ADN, ni la relación PCE/(PCE+NCE), un indicador de toxicidad para células de la médula ósea, en las ratas macho y hembra; sin embargo, los 28 días de dosis orales repetidas de nanopartículas de plata indujeron toxicidad hepática y tuvieron un efecto de coagulación en la sangre periférica (Kim et al., 2008). Los resultados del presente estudio muestran, que la viabilidad celular de los FGH fue superior para las Ag NP's sin adhesivo en todas las diluciones seriadas (mostrando no citotoxicidad) excepto en la dosis más alta, en donde la viabilidad celular fue similar a la del adhesivo con Ag NP's al 10, 5 y 0\%. Lo cual es indicativo de que el adhesivo MIP ortodóncico en sí mismo exhibe una citotoxidad moderada a se-

FIGURA 4. Diagrama ilustrativo de los posibles mecanismos de acción de las nanopartículas sobre las células.

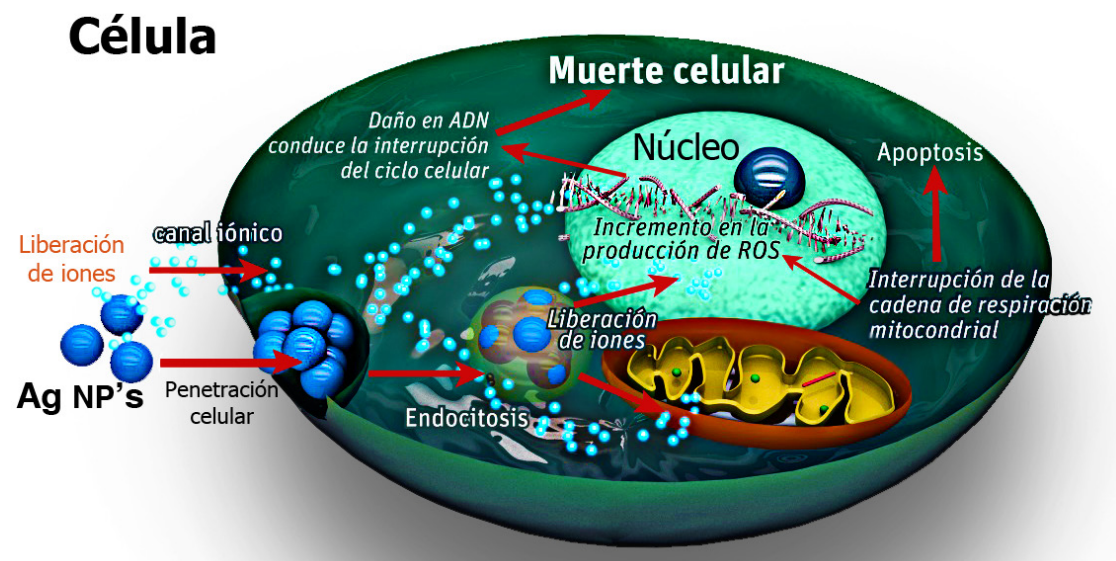

Fuente: Elaboración de los autores. 
vera similar al adhesivo si se le incorporan Ag NP's, es notorio que estadísticamente hubo diferencias significativas entre el adhesivo con nanopartículas de plata 10 en comparación con el adhesivo sin nanopartículas. La mayor viabilidad celular se observa en las nanopartículas de plata presentando diferencias estadísticamente significativas, en todas las dosis, respecto al adhesivo con o sin nanopartículas, por lo cual parece evidente que dichas nanopartículas no potencializan el efecto citotóxico del adhesivo en sí mismo.

\section{Conclusiones}

A partir de los resultados obtenidos y bajo las condiciones de este estudio es posible concluir que las bacterias en estudio, $S$. aureus, E. coli y E. faecalis fueron susceptibles tanto a las Ag NP's como al Adhesivo MIP ortodóncico adicionado con Ag NP's a diferentes concentraciones. El efecto antibacteriano de las nanopartículas de plata es dosis-dependiente. La citotoxicidad del adhesivo MIP ortodóncico en sí mismo exhibe una citotoxidad moderada a severa, mostrando que las nanopartículas de plata no potencializan el efecto citotóxico del adhesivo.

\section{Referencias}

Agarwal, N., Agarwal, U., Katiyar, S. y Gupta, S. (2015). An in vivo comparison of bacterial colonization with orthodontic bracket system. Advances In Human Biology, 5(2): 72-77, Citeseer.

Anand, M. K., Majumder, K., Venkateswaran, S. y Krishnaswamy, N. R. (2014). Comparison of shear bond strength of orthodontic brackets bonded using two different hydrophilic primers: an in vitro study. Indian Journal of Dental Research, 25(2): 191, Medknow Publications.

Argueta-Figueroa, L., Scougall-Vilchis, R. J., Morales-Luckie, R. A. y Olea-Mejía, O. F. (2015). An evaluation of the antibacterial properties and shear bond strength of copper nanoparticles as a nanofiller in orthodontic adhesive. Australian orthodontic journal, 31(1).

Balouiri, M., Sadiki, M. y Ibnsouda, S. K. (2016). Methods for in vitro evaluating antimicrobial activity: A review. Journal of Pharmaceutical Analysis, 6(2): 71-79, Elsevier. https://doi.org/10.1016/j.jpha.2015.11.005

Choi, O., Deng, K. K., Kim, N.-J., Ross Jr, L., Surampalli, R. Y. y Hu, Z. (2008). The inhibitory effects of silver nanoparticles, silver ions, and silver chloride colloids on microbial growth. Water research, 42(12): 3066-3074, Elsevier.

Choi, O. y Hu, Z. (2008). Size dependent and reactive oxygen species related nanosilver toxicity to nitrifying bacteria. Environmental science y technology, 42(12): 4583-4588, ACS Publications. https://doi.org/10.1016/j.watres.2008.02.021

Deng, S., Chung, K. H., Chan, D. C. N. y Spiekerman, C. (2016). Evaluation of bond strength and microleakage of a novel metal-titanate antibacterial agent. OPERATIVE DENTISTRY, 41(3), E48-E56. https://doi.org/10.2341/14-257-L 
Franci, G., Falanga, A., Galdiero, S., Palomba, L., Rai, M., Morelli, G. y Galdiero, M. (2015). Silver nanoparticles as potential antibacterial agents. Molecules, pp. 8856-8874. https://doi.org/10.3390/molecules20058856

García-Contreras, R., Argueta-Figueroa, L., Mejía-Rubalcava, C., Jiménez-Martínez, R., Cuevas-Guajardo, S., Sánchez-Reyna, P. A. y Mendieta-Zeron, H. (2011). Perspectives for the use of silver nanoparticles in dental practice. International Dental Journal, 61(6). https://doi.org/10.1111/j.1875-595X.2011.00072.x

Grandhi, R. K., Combe, E. C. y Speidel, T. M. (2001). Shear bond strength of stainless steel orthodontic brackets with a moisture-insensitive primer. American Journal of Orthodontics and Dentofacial Orthopedics, 119(3): 251-255, Elsevier. https://doi.org/10.1067/mod.2001.110988

Hajipour, M. J., Fromm, K. M., Ashkarran, A. A., De Aberasturi, D. J., De Larramendi, I. R., Rojo, T., Serpooshan, V., Parak, W. J. y Mahmoudi, M. (2012). Antibacterial properties of nanoparticles. Trends in biotechnology, 30(10): 499-511, Elsevier. https://doi.org/10.1016/j.tibtech.2012.06.004

Heymann, G. C. y Grauer, D. (2013). A contemporary review of white spot lesions in orthodontics. Journal of Esthetic and Restorative Dentistry, 25(2): 85-95, Wiley Online Library. https://doi.org/10.1111/jerd.12013

Hussain, S. M., Hess, K. L., Gearhart, J. M., Geiss, K. T. y Schlager, J. J. (2005). In vitro toxicity of nanoparticles in BRL 3A rat liver cells. Toxicology in vitro, 19(7): 975983, Elsevier. https://doi.org/10.1016/j.tiv.2005.06.034

Jorgensen, J. H. y Turnidge, J. D. (2015). Susceptibility test methods: dilution and disk diffusion methods. Manual of Clinical Microbiology, eleventh ed., American Society of Microbiology, 1253-1273.

Kim, A.-S., Chae, C.-H., Kim, J., Choi, J.-Y., Kim, S.-G. y Baciut, G. (2012). Silver nanoparticles induce apoptosis through the Toll-like receptor 2 pathway. Oral Surgery Oral Medicine Oral Pathology Oral Radiology, 113(6): 789-798. https://doi.org/10.1016/j.oooo.2012.01.019

Kim, Y. S., Kim, J. S., Cho, H. S., Rha, D. S., Kim, J. M., Park, J. D., Choi, B. S., Lim, R., Chang, H. K. y Chung, Y. H. (2008). Twenty-eight-day oral toxicity, genotoxicity, and gender-related tissue distribution of silver nanoparticles in SpragueDawley rats. Inhalation toxicology, 20(6): 575-583, Taylor y Francis. https://doi.org/10.1080/08958370701874663

Li, Y., Chen, D. H., Yan, J., Chen, Y., Mittelstaedt, R. A., Zhang, Y., Biris, A. S., Heflich, R. H. y Chen, T. (2012). Genotoxicity of silver nanoparticles evaluated using the Ames test and in vitro micronucleus assay. Mutation Research/Genetic Toxicology and Environmental Mutagenesis, 745(1): 4-10, Elsevier.

https://doi.org/10.1016/j.mrgentox.2011.11.010

Littlewood, S. J., Mitchell, L., Greenwood, D. C., Bubb, N. L. y Wood, D. J. (2000). Investigation of a hydrophilic primer for orthodontic bonding: an in vitro study. Journal of Orthodontics, 27(2): 181-186, Taylor y Francis.

https://doi.org/10.1093/orto/27.2.181

Matuschek, E., Brown, D. F. J. y Kahlmeter, G. (2014). Development of the EUCAST disk diffusion antimicrobial susceptibility testing method and its implementa- 
tion in routine microbiology laboratories. Clinical Microbiology and Infection, 20(4): O255-O266, Elsevier. https://doi.org/10.1111/1469-0691.12373

Miller, R. E. y Rose, S. B. (1939). Studies with the Agar Cup-Plate Method: I. A Standardized Agar Cup-Plate Technique. Journal of bacteriology, 38(5): 525-537, American Society for Microbiology (ASM).

Prabhu, S. y Poulose, E. K. (2012). Silver nanoparticles: mechanism of antimicrobial action, synthesis, medical applications, and toxicity effects. International Nano Letters, 2(1): 32, Springer. https://doi.org/10.1186/2228-5326

Solomon, S. D., Bahadory, M., Jeyarajasingam, A. V., Rutkowsky, S. A. y Boritz, C. (2007). Synthesis and study of silver nanoparticles. J. Chem. Educ., 84(2): 322, ACs Publications. https://doi.org/10.1021/ed084p322

Souza, T. A. J., Franchi, L. P., Rosa, L. R., Da Veiga, M. A. M. S. y Takahashi, C. S. (2016). Cytotoxicity and genotoxicity of silver nanoparticles of different sizes in CHO-K1 and CHO-XRS5 cell lines. Mutation Research/Genetic Toxicology and Environmental Mutagenesis, 795: 70-83, Elsevier. https://doi.org/10.1016/j.mrgentox.2015.11.002

Sudjalim, T. R., Woods, M. G. y Manton, D. J. (2006). Prevention of white spot lesions in orthodontic practice: a contemporary review. Australian Dental Journal, 51(4): 284-289, Wiley Online Library.

Sukontapatipark, W., El-Agroudi, M. A., Selliseth, N. J., Thunold, K. y Selvig, K. A. (2001). Bacterial colonization associated with fixed orthodontic appliances. A scanning electron microscopy study. The European Journal of Orthodontics, 23(5): 475-484, Oxford University Press.

Van Dong, P., Ha, C. H. y Kasbohm, J. (2012). Chemical synthesis and antibacterial activity of novel-shaped silver nanoparticles. International Nano Letters, 2(1): 9, Springer. https://doi.org/10.1186/2228-5326-2-9

Webster, M. J., Nanda, R. S., Duncanson, M. G., Khajotia, S. S. y Sinha, P. K. (2001). The effect of saliva on shear bond strengths of hydrophilic bonding systems. American Journal of Orthodontics and Dentofacial Orthopedics, 119(1): 54-58, Elsevier. https://doi.org/10.1067/mod.2001.109888

Xiu, Z., Zhang, Q., Puppala, H. L., Colvin, V. L. y Alvarez, P. J. J. (2012). Negligible particle-specific antibacterial activity of silver nanoparticles. Nano letters, 12(8): 4271-4275, ACS Publications. https://doi.org/10.1021/nl301934w 